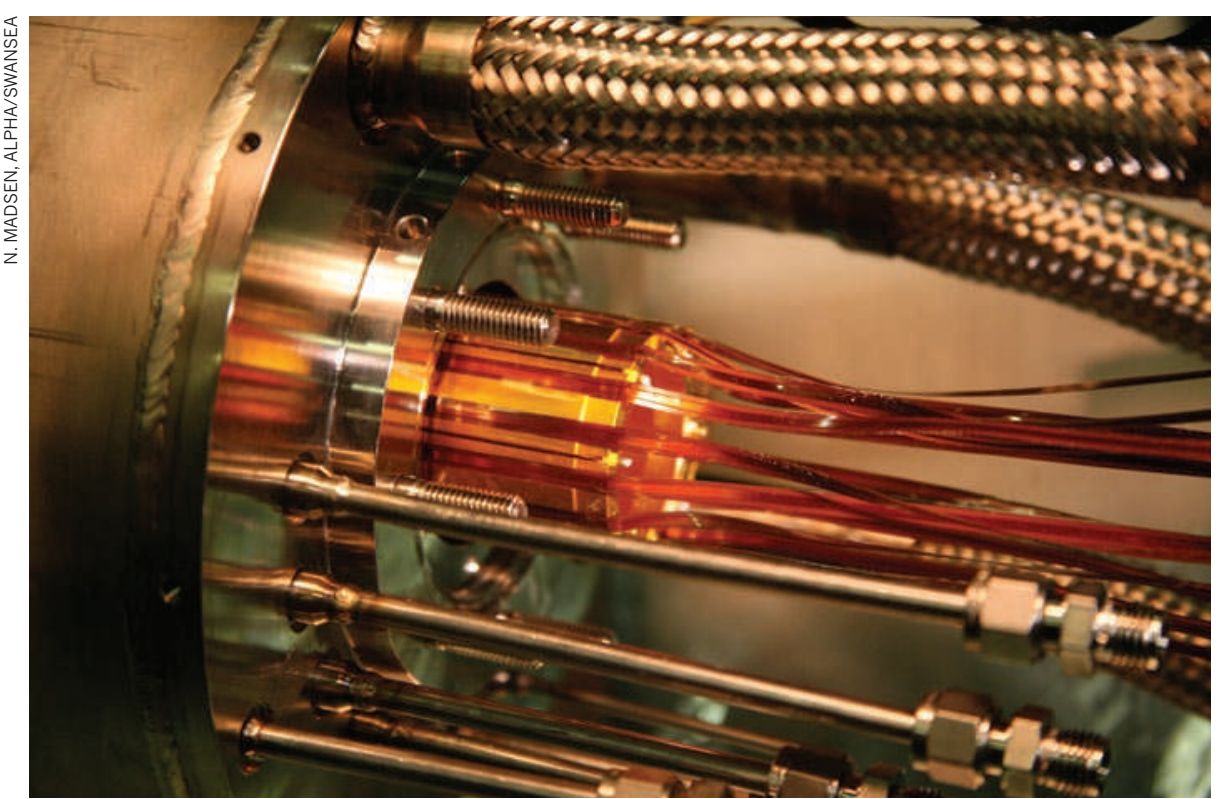

The electrodes (gold) of the trap used to combine positrons and antiprotons to form antihydrogen.

PARTICLE PHYSICS

\title{
Antimatter held for questioning
}

\section{Magnetically trapped atoms could test fundamental physics.}

\section{BY EUGENIE SAMUEL REICH}

$\mathrm{F}$ Tor physicists, a bit of antimatter is a precious gift indeed. By comparing matter to its counterpart, they can test fundamental symmetries that lie at the heart of the standard model of particle physics, and look for hints of new physics beyond. Yet few gifts are as tricky to wrap. Bring a particle of antimatter into contact with its matter counterpart and the two annihilate in a flash of energy.

Now a research collaboration at CERN, Europe's particle-physics lab near Geneva, Switzerland, has managed, 38 times, to confine single antihydrogen atoms in a magnetic trap for more than 170 milliseconds. The group reported the result in Nature online on 17 November1. "We're ecstatic. This is five years of hard work," says Jeffrey Hangst, spokesman for the ALPHA collaboration at CERN.

An antihydrogen atom is made from a negatively charged antiproton and a positively charged positron, the antimatter counterpart of the electron. The objective - both for ALPHA and for a competing CERN experiment called ATRAP - is to compare the energy levels in antihydrogen with those of hydrogen, to confirm that antimatter particles experience the same electromagnetic forces as matter particles, a key premise of the standard model. "The goal is to study antihydrogen and you can't do it without trapping it," says Cliff Surko, an antimatter researcher at the University of California, San Diego. "This is really a big deal."

The ALPHA claim is the first major advance since the creation of thousands of antihydrogen atoms in 2002 by a forerunner experiment called ATHENA ${ }^{2}$ and by ATRAP $^{3}$ (see 'A brief history of antimatter'). Both experiments combined decelerated antiprotons with positrons at CERN to produce antihydrogen atoms. But, within several milliseconds, the atoms annihilated with the ordinary matter in the walls of their containers.

To prevent that from happening, the ALPHA team formed antihydrogen atoms in a magnetic trap. Although not electrically charged like antiprotons and positrons, antihydrogen — like hydrogen - has a more subtle magnetic character that arises from the spins of its constituent particles. The ALPHA researchers used an octupole magnet, produced by the current flowing in eight wires, to create a magnetic field that was strongest near the walls of the trap, falling to a minimum at the centre, causing the atoms to collect there. To trap just 38 atoms, the group had to run the experiment 335 times. "This was ten thousand times more difficult" than creating untrapped antihydrogen atoms, says Hangst - ATHENA made an estimated 50,000 of them in one go in 2002. To do spectroscopic measurements, Surko estimates that up to 100 antihydrogen atoms may need to be trapped at once.

ATRAP still hopes to reach that goal first. In a paper due out in Physical Review Letters, the collaboration reports that it has efficiently separated antiprotons from the cold electrons that are used to cool them down, a step towards creating slower-moving antihydrogen atoms that might stay trapped for longer. "Rather than trying to demonstrate that we can confine 38 antihydrogen atoms for a small fraction of a second, we are working on new methods to produce and trap much larger numbers of colder atoms," says Gerald Gabrielse, ATRAP's spokesman. "We shall see which approach is more fruitful."

Two other collaborations aim to study antihydrogen. In 2003, the international ASACUSA experiment at CERN proposed a scheme to create a beam of antihydrogen atoms ${ }^{4}$. Yasunori Yamazaki, an atomic physicist at the Advanced Science Institute in Saitama, part of Japan's RIKEN network of research labs, now says the group has produced such a beam and may be able to use it to study the energy levels in antihydrogen without needing to trap the atoms. Another CERN experiment called AEgIS is starting to compare the effect of gravity on antihydrogen with that on ordinary hydrogen. Antimatter is almost certain to fall at the same rate as normal matter, but if it doesn't the results could help scientists to distinguish between alternative approaches to unifying quantum theory with general relativity. -

1. Andresen, G. B. et al. Nature advance online publication doi:10.1038/nature09610 (2010).

2. Amoretti, M. et al. Nature 419, 456-459 (2002)

3. Gabrielse, G. et al. Phys. Rev. Lett. 89, 213401 (2002)

4. Mori, A. \& Yamazaki, Y. Europhys. Lett. 63, 207-213 (2003).

\section{A BRIEF HISTORY OF ANTIMATTER}

\begin{tabular}{|l|l|l|}
\hline 1931 & 1932 & 1955 \\
\hline $\begin{array}{l}\text { Paul Dirac } \\
\text { predicts the } \\
\text { existence of } \\
\text { antimatter. }\end{array}$ & $\begin{array}{l}\text { Positron } \\
\text { discovered at the } \\
\text { California Institute } \\
\text { of Technology. }\end{array}$ & $\begin{array}{l}\text { Antiproton discovered } \\
\text { at Lawrence Berkeley } \\
\text { National Laboratory } \\
\text { (LBNL) in California. }\end{array}$ \\
\hline
\end{tabular}

\section{6}

\section{5}

Antineutron discovered by scientists at the LBNL.
Antideuteron (an antiproton and an antineutron) created at CERN near Geneva, Switzerland, and at Brookhaven National Laboratory in New York

\begin{tabular}{|l|l|l|}
\hline 1995 & 2002 & $\mathbf{2 0 1 0}$ \\
\hline $\begin{array}{l}\text { Observations } \\
\text { of anti- } \\
\text { hydrogen } \\
\text { at CERN. }\end{array}$ & $\begin{array}{l}\text { Creation of } \\
\text { thouands of } \\
\text { antihydrogen } \\
\text { atoms at CERN. }\end{array}$ & $\begin{array}{l}38 \text { atoms of } \\
\text { antihydrogen } \\
\text { trapped at } \\
\text { CERN. }\end{array}$ \\
\hline
\end{tabular}

